\title{
Preliminary investigation of supercritical- fluid-assisted nebulization for enhanced response in electrospray mass spectrometry
}

\author{
T.R. Baker ${ }^{1}$ and J.D. Pinkston ${ }^{2}$ \\ ${ }^{1}$ Procter \& Gamble Pharmaceuticals, Health Care Research Center, PO Box 8006, Mason, OH 45040-8006, USA \\ ${ }^{2}$ The Procter \& Gamble Company, Miami Valley Laboratories, PO Box 538707, Cincinnati, OH 45253-8707, USA
}

\begin{abstract}
A preliminary investigation of the use of supercritical-fluid (SF) $\mathrm{CO}_{2}$ to assist nebulization in electrospray HPLC/MS has been conducted. When used in conjunction with a nebulizing gas and a separately applied drying gas, a significant benefit was derived from the use of $\mathrm{CO}_{2}$. The sensitivity enhancement from the SF-assistance was more significant for flow rates above $0.2 \mathrm{ml} / \mathrm{min}$ with highly-aqueous mobile-phase compositions. For example, using a $0.5 \mathrm{ml} / \mathrm{min}$ flow-rate ( $85 \%$ aqueous), a seven-fold increase in peak height and area was observed with SFassistance.
\end{abstract}

Keywords: LC/MS, assisted nebulization, assisted electrospray, electrospray

\section{Introduction}

The electrospray technique is a well-known means of interfacing high-performance liquid chromatography (HPLC) with mass spectrometry (MS). Electrospray HPLC/MS interfaces transfer a fraction of the analyte from the liquid phase to the gas phase in an ionized form, allowing sensitive detection by a mass spectrometer. An integral step in all theoretical treatments of electrospray is rapid evaporation of the tiny droplets produced in the spray $[1,2]$.
In its purest form (unassisted) electrospray operates most effectively at low $\mu \mathrm{l} / \mathrm{min}$ flow rates [1]. However, most HPLC techniques operate at far higher flow rates, requiring either effluent splitting or flow-rate reduction, as in capillary HPLC, to be compatible with "unassisted" electrospray ionization. A number of means of assisting the electrospray process have been devised to allow the effective use of higher flow rates. Prominent among these is pneumaticallyassisted electrospray or IonSpray ${ }^{\circledast}$. As the name implies, this version uses a concentric stream of gas (nitrogen or air) to assist the nebulization process [3]. This allows the effective use of flow rates from the tens of $\mu \mathrm{l} / \mathrm{min}$ to approximately $200 \mu \mathrm{l} / \mathrm{min}$. A further enhancement of this approach is termed TurboIonSpray ${ }^{\circledR}$. This involves the addition of a second stream of gas that is heated and directed perpendicularly to the eluent spray plume, between the sprayer tip and the vacuum orifice [4]. This permits effective use of HPLC flow rates from hundreds of $\mu 1 / \mathrm{min}$ up to $1 \mathrm{ml} / \mathrm{min}$.

Other means of assisting the electrospray desolvation/ionization process are also widely utilized. Electrospraying eluent through a heated capillary tube [5,6], a multi-channel tube, or the so-called Z-spray ${ }^{\circledR}[7,8]$ has been used as a means of separating the ambient-pressure electrospray region from the lower-pressure region of the mass spectrometer. These arrangements are also assisted by a nebulizing gas and allow flow rates up to about $1 \mathrm{ml} / \mathrm{min}$. Other less-widely-utilized means of assisting the electrospray process have also been reported. These include thermallyassisted electrospray [9] and ultrasonic nebulization [10]. 
During our development of a modified electrospray interface for open-tubular [11] and then packed-column [12] supercritical-fluid chromatography/mass spectrometry (otSFC/MS and pcSFC/MS, respectively), we noted analyte response exceeding our expectations. We speculated that the enhanced response might be due to an improved spray plume (finer droplets) caused by a mobile phase consisting primarily of $\mathrm{CO}_{2}$ with a lesser amount of polar organic solvent such as methanol, an essentially "self-nebulizing" mobile phase. One aspect of this unique nebulization was the appearance of the spray-cone formed by the SFC effluent in the pneumatically-assisted electrospray interface. We had previously made the empirical observation that one factor in obtaining strong and steady signal for an HPLC/IonSpray-MS experiment was a "proper" spray-cone. Specifically, a fine, symmetrical, and straight spray-cone produced the best results. The spray-cone produced by our $\mathrm{pcSFC} / \mathrm{MS}$ interfacing effort visually appeared to be superior to any we had ever produced during HPLC/MS experimentation.

These observations led us to conduct a preliminary investigation of the addition of supercritical $\mathrm{CO}_{2}$ upstream of the nebulization zone in the conventional IonSpray interface (with TurboIonSpray gun) operating with HPLC eluents. Analytes were injected in the flow-injection-analysis (FIA) mode using reversed-phase HPLC (aqueous) eluents. This limited investigation suggests that some benefit may be derived from supercritical-fluid (SF)-assisted HPLC/IonSpray-MS.

\section{Experimental}

\section{HPLC (FIA system)}

The FIA flow was provided by Waters 600MS pumps (Milford, MA, USA). The FIA flow was connected to the "sheath-flow" inlet of the interface (Fig. 1), as originally designed for SFC/MS.

\section{Supercritical $\mathrm{CO}_{2}$ delivery}

The supercritical $\mathrm{CO}_{2}$ was introduced, as described below, using a Model 260D syringe pump from Isco (Lincoln, NE, USA) operated under pressure control. The cylinder of the pump was equipped with a cooling jacket and was cooled to approximately $10{ }^{\circ} \mathrm{C}$ using a Model RTE-111 circulating chiller from Neslab (Portsmouth, NH, USA). This ensured that the pump was efficiently filled with, and pumped, liquid $\mathrm{CO}_{2}$. While the pump delivered a liquid, the $\mathrm{CO}_{2}$ was heated beyond its critical temperature $\left(31.08{ }^{\circ} \mathrm{C}\right)$ in the IonSpray interface (with TurboIonSpray gun on). We previously reported that the temperature of the components inside the IonSpray interface rises to $\sim 60{ }^{\circ} \mathrm{C}$ during operation [11] in this mode.

\section{Mass spectrometry}

The experiments were conducted using a Model API III+ tandem quadrupole mass spectrometer from PE-Sciex

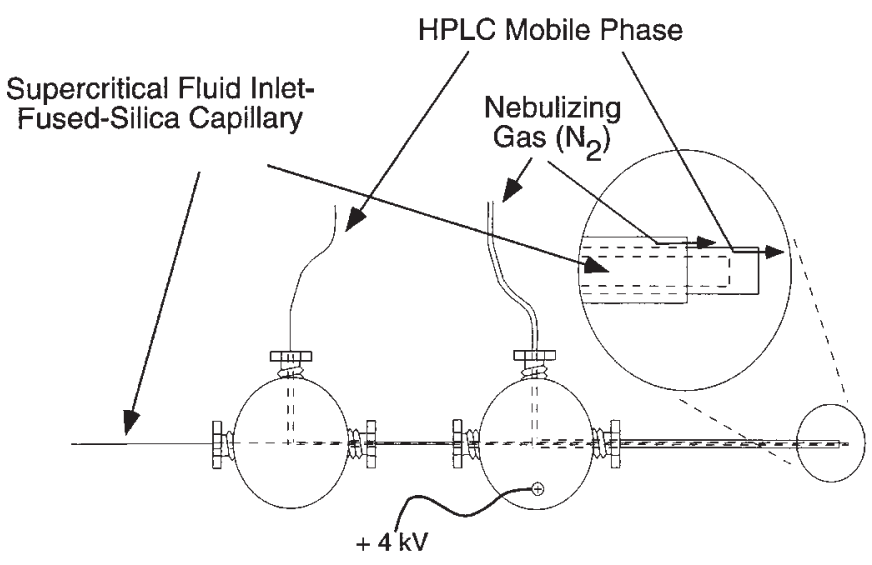

Figure 1. Modified lonSpray interface used for SF-assisted nebulization HPLC/MS.

(Concord, Ontario, Canada). The same ultra-high purity $\mathrm{N}_{2}$ source (liquid $\mathrm{N}_{2}$ boil-off) was used for curtain, nebulizer, and TurboIonSpray gases. The sprayer potential was held at $4 \mathrm{kV}$. Values used for the orifice ring voltage $(45 \mathrm{~V})$, curtain gas flow (1.4 liter/min), TurboIonSpray gun parameters (8 liter/min, $500{ }^{\circ} \mathrm{C}$ ), nebulizer gas pressure (46 psi, $0.32 \mathrm{MPa})$, and interface plate temperature $\left(55^{\circ} \mathrm{C}\right)$ were typical of conventional HPLC/MS experiments. Values were as indicated by the instrument. The mass spectrometer was tuned in the conventional manner with an aqueous solution of poly(propylene glycol) (PPG) $\left(1 \times 10^{-4} \mathrm{M}\right.$ PPG 1000 , $2 \times 10^{-4}$ M PPG 2000, $1 \times 10^{-3} \mathrm{M}$ ammonium acetate) using the unaltered IonSpray interface. Direct infusion of the tuning solution was accomplished using a Model 22 syringe pump from Harvard Apparatus (South Natick, MA, USA) set at $10 \mu \mathrm{l} / \mathrm{min}$.

\section{Modified lonSpray interface}

The IonSpray interface was modified for use as a pcSFC/MS interface, as previously described [12]. For these experiments we used the modified interface, as shown in figure 1. The liquid $\mathrm{CO}_{2}$ was introduced through a fused-silica transfer line (deactivated fused-silica, 50- $\mu \mathrm{m}$ ID, 200-mm OD, Dionex, Sunnyvale, CA, USA). The transfer line extended through both tees and the stainless-steel tubing (see below) to the tip of the sprayer. The FIA or HPLC mobile phase entered the first tee (left in diagram). This liquid ran coaxially to the fused-silica $\mathrm{CO}_{2}$ line through a $0.41-\mathrm{mm}(0.016-$ in.) ID, 0.71-mm (0.028-in.) OD, 11.4-cm long, stainlesssteel capillary (Small Parts Inc., Boca Raton, FL, USA). The mobile phase flowed in the space between the stainless-steel capillary and fused-silica transfer line and did not interact with the supercritical $\mathrm{CO}_{2}$ until both reached the tip of the spray device. The nebulizer gas was added at the sprayer tee (right in figure 1), in the conventional manner. This gas flowed coaxially to the HPLC mobile phase and to the 
supercritical $\mathrm{CO}_{2}$ through a $0.84-\mathrm{mm}$ (0.033-in.) ID, 1.27-mm (0.050-in.) OD, stainless-steel nebulizer tube. The fused-silica and stainless-steel capillary connections to the tees employed graphite-filled-Vespel capillary ferrules (Valco Instruments, Houston, TX, USA) or PEEK fittings (Upchurch Scientific, Oak Harbor, WA, USA). The stainlesssteel mobile-phase capillary protruded past the end of the stainless-steel nebulizer-gas tube by approximately $1 \mathrm{~mm}$. While the flow-path of the mobile phase was not optimized for low extra-column volume, this arrangement did serve to test the effect of SF assistance.

\section{Upstream $\mathrm{CO}_{2}$ addition}

The HPLC effluent and liquid $\mathrm{CO}_{2}$ were mixed approximately $30 \mathrm{~cm}$ from the standard API-III+ IonSpray sprayer tee using a low-dead-volume, stainless-steel, 1/16-inch Valco tee. The mixed effluent flowed from the mixing tee to the spray device through $50-\mu \mathrm{m}$ ID, $150-\mu \mathrm{m}$ OD fused-silica tubing.

\section{Materials and sample preparation}

\section{Solvents and samples}

All solvents were HPLC grade or better (J. T. Baker, Phillipsburg, NJ, USA). Carbon dioxide was SFC/SFE grade (Air Products, Allentown, PA, USA). Solutions of the analytes were prepared by dissolution in methanol. Norepinephrine was obtained from Lancaster Synthesis (Windham, NH, USA). The quinolonyl-lactam antibacterial (QLA) was synthesized in-house.

\section{Mobile phases}

The mobile phase for the norepinephrine analyses consisted of $85 \%(\mathrm{v} / \mathrm{v}) 10-\mathrm{mM}$ heptafluorobutyric acid in water with $15 \%$ methanol. The water was deionized and purified with a Milli-Q Reagent Water System (Millipore, Bedford, MA, USA). For the QLA, the mobile phase was a 34/66 mixture of water/methanol with $0.1 \%$ formic acid and $2 \mathrm{mM}$ ammonium acetate overall.

\section{Results and discussion}

Our ultimate goal was to investigate the utility of $\mathrm{SF} \mathrm{CO}_{2}$ assisted HPLC/MS/MS as a means of improving sensitivity for the analysis of pharmaceutical compounds. For this work, we typically use reversed-phase eluents and 2-mm ID, 5 to $15-\mathrm{cm}$ long HPLC columns. Due to the specificity of the tandem mass spectrometry detection scheme (selected reaction monitoring or SRM), these separations are typically rapid and use eluent flow-rates between $200 \mu \mathrm{l} / \mathrm{min}$ and $500 \mu \mathrm{l} / \mathrm{min}$. The first compound we tested was norepinephrine. The L-form of this drug is used therapeutically as an adrenergic (vasoconstrictor) agent. FIA of this compound, using the aqueous mobile phase described above and monitoring the collisionally-induced dissociation (CID) reaction $\mathrm{m} / \mathrm{z} 170 \rightarrow \mathrm{m} / \mathrm{z}$ 107, was conducted using the modified interface shown in figure 1 , but without $\mathrm{SF} \mathrm{CO}_{2}$ addition. A reproducible FIA profile was obtained in this manner. The typical example displayed in figure 2 (top) resulted from a $50-\mu \mathrm{l}$ injection of $73 \mathrm{ng}$ of norepinephrine (eluent flow $500 \mu \mathrm{l} / \mathrm{min}$ ). Within minutes this experiment was repeated. The only difference was that $\mathrm{SF} \mathrm{CO}_{2}$ flow was added. With the $\mathrm{SF} \mathrm{CO}_{2}$ transfer line described above, $20 \mathrm{MPa}$ provided approximately $2.0 \mathrm{ml} / \mathrm{min}$ fluid flow, or $\sim 2.0 \mathrm{~g} / \mathrm{min}$, resulting in an expanded gas flow of $\sim 1.2$ liter/min in the source. The result with SF-assisted nebulization is shown in the lower portion of figure 2. A better than 7-fold signal enhancement was obtained with the SF-assistance, based on either peak height or area.

A similar experiment was conducted with a much larger amount of a different analyte, using a different mobile phase. QLAs have been investigated as antiinfective agents. We had developed an HPLC/MS/MS assay for one of these compounds that required an isocratic aqueous mobile phase as described above. Using a $500-\mu 1 / \mathrm{min}$ flow of this aqueous mobile phase, FIA with MS/MS detection was performed with and without SF-assisted nebulization. The results for

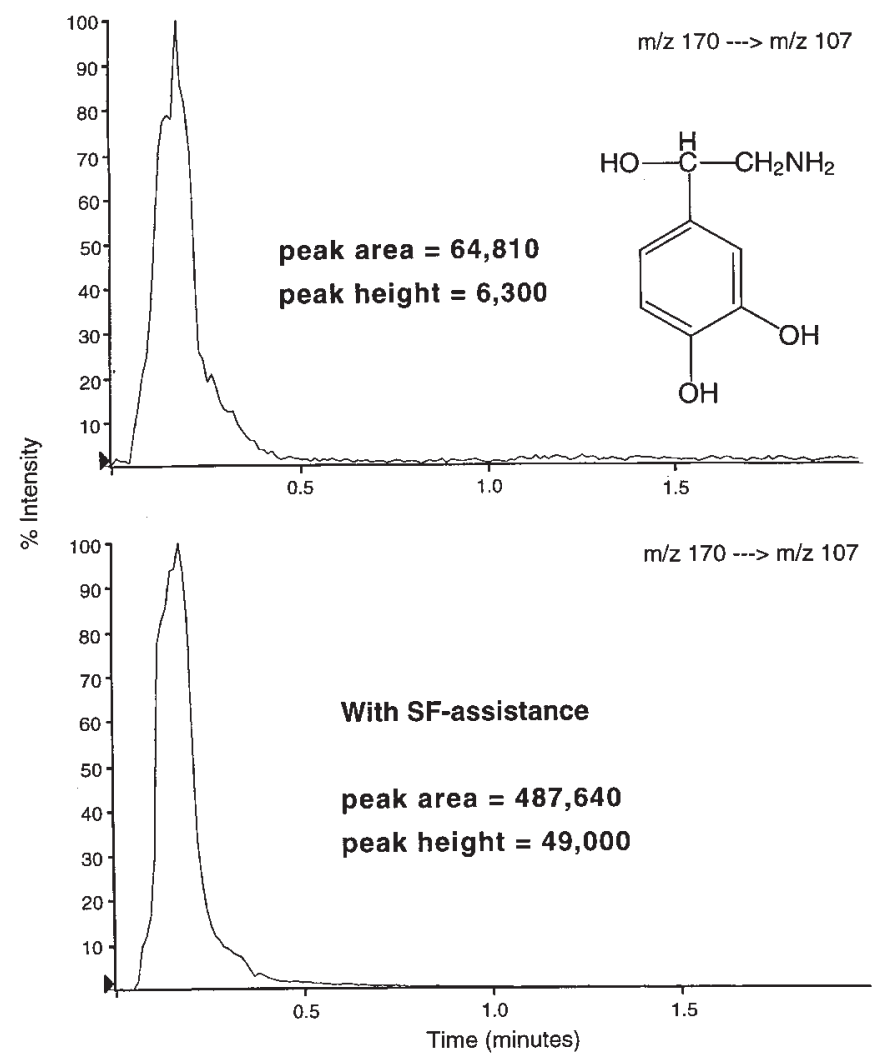

Figure 2. Flow-injection profiles of $73 \mathrm{ng}$ of norephinephrine without (top) and with (bottom) SF-assisted nebulization. At this $0.5 \mathrm{ml} / \mathrm{min}$ flow-rate (85\% aqueous), over a seven-fold increase in peak height or area was observed with SF-assistance. 
1.5-mg FIA injections are displayed in figure 3. Note that the instrument was not optimized for detection of this compound and it appeared to be absorbing onto the tubing walls (i.e., peak tailing). In this case, a better than 2 -fold benefit (peak height or area) was obtained. It's important to note that we attempted to repeat this FIA experiment using a mobile-phase flow rate of $1 \mathrm{ml} / \mathrm{min}$. The SF-assisted leg of the experiment proceeded as expected and provided results equivalent to those shown in the lower portion of figure 3. However, we were not able to complete the unassisted (no $\mathrm{CO}_{2}$ addition) leg of the experiment, because the spray-cone was not stable, and we felt the amount of liquid on the walls of the source and near the vacuum orifice was excessive.

We believe that the differences in benefits obtained using the SF-assisted nebulization in these two cases are a function of mobile-phase composition and are not related to analyte differences. The first eluent contained $85 \%$ water, while the second only contained $34 \%$. We also observed that the benefit due to SF-assisted nebulization decreased noticeably as we reduced the mobile phase flow-rate (i.e., from $1 \mathrm{ml} / \mathrm{min}$ to $200 \mu \mathrm{l} / \mathrm{min}$ ). These observations are not surprising and are analogous to the benefits derived from the use of the TurboIonSpray gun in pneumatically-assisted electrospray. The benefits derived from the heated nitrogen stream applied orthogonally to the spray-cone clearly increase as mobile-phase flow rate and aqueous content increase. One must also consider that addition of $\mathrm{CO}_{2}$ to aqueous mobile phases will result in the formation of carbonic acid, with a concomitant drop in $\mathrm{pH}$. We have observed that the $\mathrm{pH}$ of the aqueous phase in a liquid $\mathrm{CO}_{2}$ /water mixture at a pressure of approximately $8 \mathrm{MPa}$ drops rapidly to approximately 4 [13]. This effect may also affect ionization efficiency in pneumatically-assisted electrospray desorption/ionization.

\section{Optimization of $\mathrm{CO}_{2}$ pressure}

A rudimentary optimization of the $\mathrm{CO}_{2}$ delivery pressure was conducted. This work was performed using FIA of norepinephrine, as described earlier. Raising the $\mathrm{CO}_{2}$ delivery pressure to $20 \mathrm{MPa}$ resulted in a $50 \%$ improvement in response when compared to $10 \mathrm{MPa}$. With the transfer-line configuration and temperatures described above, these pressures correspond to flows of liquid $\mathrm{CO}_{2}$ from the pump of $2.3 \mathrm{~g} / \mathrm{min}(\sim 1.4 \mathrm{liter} / \mathrm{min}$ expanded gas in the source) and $1.7 \mathrm{~g} / \mathrm{min}$ ( 1.0 liter/min expanded gas), respectively. At a pressure of $40 \mathrm{MPa}$ (flow of $3.4 \mathrm{~g} / \mathrm{min}$ or $\sim 2.0 \mathrm{liter} / \mathrm{min}$ expanded gas), the spray-cone was disrupted. This caused a dramatic decrease in signal and signal-to-noise ratio $(\mathrm{S} / \mathrm{N})$, as compared to $20 \mathrm{MPa}$.

\section{Position of the fused-silica $\mathrm{CO}_{2}$ line in the spray tip}

The position of the tip of the fused-silica $\mathrm{CO}_{2}$ capillary relative to the stainless-steel mobile-phase capillary was optimized. The position providing the best signal and $\mathrm{S} / \mathrm{N}$ was achieved by withdrawing the $\mathrm{CO}_{2}$ capillary inside the mobile-phase capillary by approximately $0.5 \mathrm{~mm}$. This position is identical to that found most suitable for SFC/MS
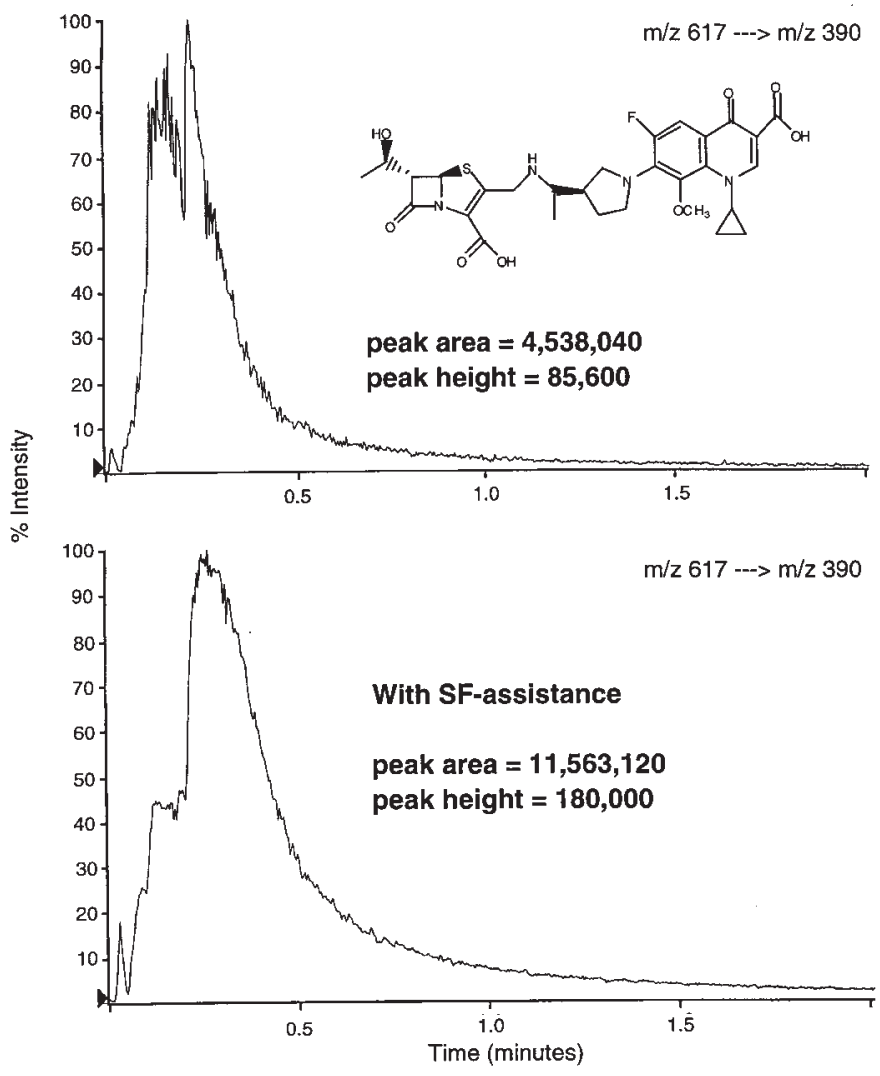

Figure 3. Flow-injection profiles of a QLA without (top) and with (bottom) SF-assisted nebulization. The improvement for this compound and mobile phase ( $34 \%$ aqueous, $0.5 \mathrm{ml} / \mathrm{min}$ ) is greater than a factor of two, based on peak height or area.

experiments [12]. Withdrawing the tip of the $\mathrm{CO}_{2}$ capillary any further inside the metal tubing resulted in lower signal and $\mathrm{S} / \mathrm{N}$.

\section{Upstream $\mathrm{CO}_{2}$ addition}

Further experiments were conducted in which the liquid $\mathrm{CO}_{2}$ and HPLC effluent streams were mixed approximately $30 \mathrm{~cm}$ from the IonSpray device, as described in the Experimental section. Note that this mixture was likely 2-phase for the more highly aqueous mobile phase. No improvement in signal or $\mathrm{S} / \mathrm{N}$ was observed over our earlier results with addition of $\mathrm{CO}_{2}$ at the sprayer tip.

\section{Conclusions}

This preliminary investigation leads us to believe that SFassisted nebulization of HPLC eluent may find utility, particularly when used with highly aqueous mobile phases at flow rates typical of $4.6-\mathrm{mm}-\mathrm{ID}$ columns (i.e., $\sim 1 \mathrm{ml} / \mathrm{min}$ ). 
It is possible that benefits beyond those we observed might be realized with an interface specifically designed for SFassisted nebulization. Furthermore, Joule-Thompson cooling of the IonSpray tip, due to the rapid expansion of the $\mathrm{CO}_{2}$, may be beneficial in the analysis of thermally-labile analytes. Finally, the substitution of other dense gases for $\mathrm{CO}_{2}$, such as $\mathrm{NH}_{3}, \mathrm{SF}_{6}$, etc., may result in benefits not observed with $\mathrm{CO}_{2}$. Ammonia, for example, may enhance ionization of acidic analytes. $\mathrm{SF}_{6}$ would likely allow the use of higher IonSpray voltages before a corona discharge occurs.

\section{Acknowledgments}

The authors wish to thank Tom Covey of PE-Sciex for his enthusiasm and encouragement of this work. We also thank Tom Chester, Mary Kay Dirr, and Tom Eichhold of Procter $\&$ Gamble for useful discussions and assistance.

\section{References}

1. Fenn, J. B.; Mann, M.; Meng, C. K.; Wong, S. F.; Whitehouse, C. M. Mass Spectrom. Rev. 1990, 9, 37-70.

2. Kebarle, P.; Tang, L. Anal.Chem. 1993, 65, 972A-86A.

3. Covey, T. R.; Bonner, R. F.; Shushan, B. I.; Henion, J. Rapid Commun. Mass Spectrom. 1988, 2, 249-56.

4. Covey, T. R.; Anacleto, J. F.; French, J. B. Development of dynamic gas focusing for pneumatically-assisted electrospray (IonSpray) sample introduction, Proceedings of the 42nd
ASMS Conference on Mass Spectrometry and Allied Topics, p. 771, May 29 - June 3, 1994, Chicago, Illinois.

5. Hail, M.; Mylchreest, I. C., Characterization of a new atmospheric-pressure ionization interface for liquid chromatography/mass spectrometry, Presented at the 41st ASMS Conference on Mass Spectrometry and Allied Topics, May 31June 4, 1993, San Francisco, CA.

6. Mylchreest, I. C.; Hail, M., A heated capillary atmosphericpressure ionization source with an atmospheric-pressure chemical ionization interface, Presented at the 41st ASMS Conference on Mass Spectrometry and Allied Topics, May 31June 4, 1993, San Francisco, CA.

7. Hughes, C. J.; Bateman, R. H.; Bordoli, R. S.; Morris, M.; Little, D.; Martin, L., A universal low-flow-rate interface for API mass spectrometry, Presented at the 46st ASMS Conference on Mass Spectrometry and Allied Topics, May 31June 4, 1998, Orlando, FL.

8. Bertrand, M.; Vayer, P.; Perez, J. C.; McDowall, M.; Preece, S., Evaluation of a novel API ion source for the high-throughtput determination of drugs directly from cell culture media for in vitro studies, Presented at the 46st ASMS Conference on Mass Spectrometry and Allied Topics, May 31-June 4, 1998, Orlando, FL.

9. Lee, E. D.; Henion, J. D. Rapid Commun. Mass Spectrom. 1992, 6, 727-33.

10. Barber, T. A.; Shen, W. L.; Whitehouse, C. M.; Fenn, J. B. Anal.Chem. 1994, 66, 406-14.

11. Pinkston, J. D.; Baker, T. R. Rapid Commun. Mass Spectrom. 1995, 9, 1087-94.

12. Baker, T. R.; Pinkston, J. D. J. Am. Soc. Mass Spectrom. 1998, 9, 498-509.

13. Pinkston, J. D. and Chester, T. L., The Procter \& Gamble Company, unpublished results, 1999. 